\title{
Comparación de la eficacia del color y sensibilidad entre el Peróxido de Hidrógeno y Peróxido de Carbamida: Revisión de la literatura
}

\author{
Comparison of Color Efficiency and Sensitivity Between Hydrogen Peroxide and Carbamide
}

Peroxide: Literature review

Comparação da eficiência e sensibilidade da cor entre o Peróxido de Hidrogênio e o Peróxido de Carbamida: Revisão da literatura

Recibido: 15/04/2021 | Revisado: 19/04/2021 | Acepto: 20/04/2021 | Publicado: 25/04/2021

\author{
Lizbeth Eliana Salazar García \\ ORCID: https://orcid.org/0000-0002-2056-1150 \\ Universidad de Cuenca, Ecuador \\ E-mail: lis.salazarg@gmail.com \\ Estefanía Alexandra González Chimbo \\ ORCID: https://orcid.org/0000-0002-7332-5821 \\ Universidad de Cuenca, Ecuador \\ E-mail: estefaniagonzalez22m@hotmail.com \\ Iván Andrés Palacios Astudillo \\ ORCID: https://orcid.org/0000-0002-5857-5347 \\ Universidad de Cuenca, Ecuador \\ E-mail: andrés.palacios@ucuenca.edu.ec
}

\begin{abstract}
Resumen
Hoy en día existen varios métodos de aplicación del peróxido de carbamida e hidrógeno para su uso en el clareamiento dental, mediante esta investigación de la literatura se trata de determinar cuál es la mejor opción. Objetivo: Comparar mediante una revisión de literatura la eficacia del color y la sensibilidad dental producida por el peróxido de hidrógeno y el peróxido de carbamida. Método: Las bases de datos empleadas fueron PUBMED, Scopus y Web of Science. El método de búsqueda fue "TOOTH WHITENING AND HYDROGEN PEROXIDE AND CARBAMIDE PEROXIDE" aplicando los criterios de inclusión y exclusión se obtuvo 9 artículos para esta revisión de la literatura. Resultado: De acuerdo a los estudios analizados se encontró que todos los agentes aclaradores mejoran el aspecto del color de las estructuras dentales. En la mayoría de los estudios la sensibilidad dental, estaba presente; sin embargo, el peróxido de carbamida produce menos sensibilidad. Conclusión: El peróxido de hidrógeno como el peróxido de carbamida son efectivos para producir un cambio de color de los dientes. En los dientes más oscuros el tratamiento es más efectivo independientemente del agente aclarante utilizado. Por otro lado, la sensibilidad es menor cuando se aplica peróxido de carbamida.
\end{abstract}

Palabras claves: Blanqueamiento dental; Peróxido de hidrógeno; Peróxido de carbamida; Sensibilidad dental.

\begin{abstract}
Nowadays there are several methods of applying carbamide and hydrogen peroxide for use in dental whitening, this literature investigation tried to determine which is the best option. Objective: To compare through a literature review the efficacy of tooth color and sensitivity produced by hydrogen peroxide and carbamide peroxide. Method: The databases used were PUBMED, Scopus and Web of Science. The search method was "TOOTH WHITENING AND HYDROGEN PEROXIDE AND CARBAMIDE PEROXIDE", applying the inclusion and exclusion criteria, 9 articles were obtained for this literature review. Result: According to the analyzed studies, it was found that all lightening agents improve the color appearance of dental structures. In most studies tooth sensitivity was present; however, carbamide peroxide produces less sensitivity.Conclusion: Hydrogen peroxide like carbamide peroxide are effective in producing a discoloration of teeth. In darker teeth the treatment is more effective regardless of the lightening agent used. On the other hand, the sensitivity is lower when carbamide peroxide is applied.
\end{abstract}

Keywords: Tooth Whitening; Hydrogen Peroxide; Carbamide Peroxide; Tooth Sensitivity.

\section{Resumo}

Atualmente existem vários métodos de aplicação de carbamida e peróxido de hidrogênio para uso no clareamento dental, por meio desta investigação na literatura procura-se determinar qual a melhor opção. Objetivo: Comparar, por meio de revisão da literatura, a eficácia da cor e da sensibilidade dentária produzida pelo peróxido de hidrogênio e peróxido de carbamida. Método: As bases de dados utilizadas foram PUBMED, Scopus e Web of Science. O método de busca foi "BRANQUEAMENTO DE DENTES E PERÓXIDO DE HIDROGÊNIO E PERÓXIDO DE CARBAMIDA", 
aplicando os critérios de inclusão e exclusão, foram obtidos 9 artigos para esta revisão de literatura. Resultado: De acordo com os estudos analisados, constatou-se que todos os agentes clareadores melhoram a coloração das estruturas dentais. Na maioria dos estudos, a sensibilidade dentária estava presente; entretanto, o peróxido de carbamida produz menos sensibilidade. Conclusão: O peróxido de hidrogênio como o peróxido de carbamida são eficazes na produção de descoloração dos dentes. Em dentes mais escuros, o tratamento é mais eficaz, independentemente do agente clareador utilizado. Por outro lado, a sensibilidade é menor quando o peróxido de carbamida é aplicado.

Palavras-chave: Branqueamento De Dentes; Peróxido De Hidrogênio; Peróxido De Carbamida; Sensibilidade Dentária.

\section{Introducción}

Actualmente existe un gran número de pacientes que requieren un tratamiento odontológico especializado para perfeccionar la apariencia de la superficie del diente, como opciones para mejorar la estética dental existen restauraciones directas e indirectas, también tratamientos no restaurativos que son mínimamente invasivos ya que no requieren la eliminación de tejido dental como es el clareamiento dental, siendo éste un método seguro y conservador (Jadhav \& Mattigatti, 2020) (Nie et al., 2017) (Kothari et al., 2020).

Los agentes más utilizados para el clareamiento dental vital son el peróxido de hidrógeno $(\mathrm{PH})$ y el peróxido de carbamida (PC) estos agentes pueden ser empleados en diferentes concentraciones (Ahrari et al., 2020)(Nie et al., 2017). El clareamiento dental se da por la descomposición de los peróxidos, produciendo radicales libres que oxidan los componentes orgánicos del tejido dental. La eficacia del clareamiento se determina principalmente por el cambio de color del diente; sin embargo, uno de los principales efectos secundarios es la sensibilidad dental con una incidencia de $51 \%$ y $63 \%$ para las técnicas de clareamiento en el hogar y consultorio, respectivamente (Peixoto et al., 2018) (Correa et al., 2016).

Los sistemas de clareamiento dental hoy en día son muy solicitados, cabe destacar que en el campo odontológico llevan muchos años siendo utilizados y estos al paso del tiempo han ido evolucionando con el objetivo de alcanzar un tratamiento dental eficaz (Solís Cessa, 2018).

En 1911 Fisher introdujo un método aceptable para el clareamiento dental con el uso del PH, en algunos casos era activada con una fuente de luz o calor (Alqahtani, 2014).

Klusmier en 1962, descubrió el efecto aclarante del PC sobre el esmalte dental cuando trataba la inflamación gingival posterior al tratamiento de ortodoncia. A finales de 1980 empieza la demanda del aclaramiento dental como tratamiento estético. Haywood y Heymann describieron la técnica en 1989, que consistía en la aplicación de PC al 10\% en una cubeta durante ocho horas en la noche (Alkahtani et al., 2020) (Sulieman et al., 2006) .

Los sistemas de clareamiento se pueden aplicar tanto en el consultorio como en el hogar. Los sistemas de clareamiento en el consultorio por lo general utilizan agentes oxidantes en altas concentraciones por periodos de tiempo cortos. Por otro lado, los sistemas de clareamiento en el hogar, se pueden subdividir en dos subgrupos: a) productos aclaradores supervisados por profesionales, los cuales consisten en el uso de un gel depositado en una cubeta; b) productos de venta libre que pueden adquirirse sin la supervisión del odontólogo, estos incluyen pastas dentales, tiras, geles, enjuagues y aclaradores dentales en bandeja, tienen un potencial aclarador bajo (Rodríguez-Martínez et al., 2019).

Los sistemas de clareamiento están compuestos por ingredientes activos e inactivos, entre los ingredientes activos se encuentra PH o PC y entre los inactivos están agentes espesantes, portadores, tensioactivos y dispersantes de pigmentos, conservantes y aromatizantes (Solís Cessa, 2018) (Alqahtani, 2014). Un agente blanqueador aclara un sustrato mediante reacciones químicas, las cuales pueden ser procesos de oxidación o reducción que implican la destrucción o modificación de grupos cromofóricos en el sustrato, así como la degradación de cuerpos de color en unidades más pequeñas y solubles que se eliminan más fácilmente en el proceso de clareamiento (Alomar \& Manubens, 2015). 
Los peróxidos tienen la capacidad de desnaturalizar proteínas, lo que aumenta el movimiento de iones a través de la estructura dental, cuando se oxidan macromoléculas que producen las pigmentaciones, estas son convertidas en dióxido de carbono y agua, que mediante difusión son removidos de la estructura dental (Moradas, 2017). El agente activo más comúnmente utilizado es el PH, debido a su bajo peso molecular y alta inestabilidad, siendo capaz de difundirse en los tejidos dentales, y descomponerse en radicales libres que actúan sobre los cromóforos (Mailart et al., 2020). El PH es una sustancia química con un enlace simple oxígeno-oxígeno, con capacidad de dañar diferentes moléculas y compuestos, puede aplicarse directamente o producirse en una reacción química a partir del PC (Rodríguez-Martínez et al., 2019). Su mecanismo de acción no es completamente conocido, este es un agente oxidante que se disocia para producir radicales libres inestables que son radicales hidroxilo (HO), radicales perhidroxilo (HOO), aniones perhidroxilo (HOO-), aniones superóxido (OO-) e iones hidrógeno, que atacarán los dobles enlaces de las moléculas pigmentadas orgánicas entre las sales inorgánicas en el esmalte dental. El cambio en la conjugación del doble enlace da como resultado componentes más pequeños y menos pigmentados, y habrá un cambio en el espectro de absorción de las moléculas de cromóforo; por tanto, se produce el clareamiento de los tejidos dentales. La reacción de los radicales con los compuestos orgánicos origina epóxidos inestables y finalmente forma alcoholes, los cuales se eliminan fácilmente por ser compuestos solubles (Alqahtani, 2014) (Solís Cessa, 2018) (Rodríguez-Martínez et al., 2019) (Eimar et al., 2012) (Eachempati et al., 2018).

El PC (CH6N2O3) es el sistema más utilizado para el clareamiento casero, está formado por PH, siendo éste su componente activo, y urea. El PH al contacto con tejidos y saliva se disocian en oxígeno y agua; la urea se descompone en amoniaco y dióxido de carbono. La urea tiene la capacidad de neutralizar el pH del medio y el amoniaco facilita la penetración del oxígeno debido a que aumenta la permeabilidad del esmalte (Solís Cessa, 2018) (Zanolla et al., 2017).

El aclaramiento con PC o PH tiene como resultado una inflamación pulpar que se caracteriza por mayor dilatación vascular, incremento en el número de macrófagos y en la respuesta inflamatoria (Vaz et al., 2016) (Luque-Martinez et al., 2016). Este proceso se debe a la presencia de defectos microscópicos a nivel del esmalte, permitiendo que el agente aclarador y sus subproductos penetren en los túbulos dentinales y lleguen a la pulpa dental, provocando una pulpitis reversible (Carlos et al., 2017). Además, el estrés oxidativo causado por la penetración del peróxido aumenta el nivel de mediadores de inflamación como prostaglandinas, bradicinina y sustancia $\mathrm{P}$, tomando en cuenta que la presencia de bradicinina es responsable del dolor dental (Rodrigues et al., 2018). Durante la reacción química que ocurre en el clareamiento dental, los tapones dentinarios formados para disminuir la sensibilidad dental, se liberan provocando un flujo de líquido interno, que excita el tejido pulpar y causa sensibilidad (Carlos et al., 2017). Si realmente es así cómo ocurre el proceso, entonces reemplazando estos tapones cuando los dientes se aclaran, antes o después del tratamiento, las sensaciones de dolor pueden alterarse mientras se logra el clareamiento dental (Radi et al., 2018) (Vaz et al., 2016) (Carlos et al., 2017).

De acuerdo a la Asociación Dental Americana (ADA), los agentes aclarantes resultan eficaces cuando el color resultante es al menos 4 tonos más claro con una guía de colores o es de 3 unidades más bajo o más alto con el sistema LAB de la Commission Internationale de l'Eclairage (CIE) (Bernardon et al., 2015). Su registro evalúa las coordenadas tridimensionales, que es un estándar utilizado a nivel mundial que asigna valores numéricos para definir el color. En este sistema los colores son expresados en 3 ejes, el eje L* indica la coordenada acromática o la luminosidad del objeto con valores de 0 (negro absoluto) a 100 (blanco absoluto). El eje $\mathrm{a}^{*}$ representa la cantidad de rojo (valor de $\mathrm{a}^{*}$ positivo), o de verde (valor de $\mathrm{a}^{*}$ negativo). El eje $\mathrm{b}^{*}$ representa la cantidad de amarillo (valor de $b^{*}$ positivo) o azul (valor de $b^{*}$ negativo). Cuando los valores de los ejes $a^{*}$ y $b^{*}$ se aproximan a cero, representan un área acromática, basada en la escala de valor (Mounika et al., 2018) (Nie et al., 2017) (Radi et al., 2018) (Eachempati et al., 2018) (Schmelling, 2016).

El color es la distribución espectral de la luz que llega al ojo cuando se ilumina un diente y se puede ver influenciado por el fondo contra el que se ven las muestras. Según la distinción del observador existe el color físico y el color perceptual, 
siendo el último el que más interesa a los pacientes. Las dimensiones perceptivas del color son la claridad, el croma y el tono. La luminosidad hace referencia al mayor o menor reflejo de la luz. El croma es el atributo por el cual los dientes parecen ser más coloridos. Los dientes se ven más rojos, amarillos o azules, por una característica denominada tono. En odontología, un aumento de la blancura se atribuye al aumento de la luminosidad y disminución del cromatismo(Pan \& Westland, 2018) .

El objetivo de este estudio fue comparar mediante una revisión de literatura la eficacia del color y la sensibilidad dental producida por peróxido de hidrógeno y peróxido de carbamida.

\section{Metodología}

Este artículo es de modelo cualitativo ya que se fundamenta en la interpretación del investigador sobre los temas analizados (Pereira et al., 2018). Esta revisión de la literatura se basa en el análisis de la bibliografía existente de los últimos 5 años, se realizó en el periodo de tiempo entre Mayo y Noviembre de 2020. Las bases de datos empleadas en la investigación fueron PUBMED, Scopus y Web of Science. Las palabras claves empleadas: TOOTH WHITENING, HYDROGEN PEROXIDE, CARBAMIDE PEROXIDE, el operador booleano aplicado en la búsqueda fue: AND. Los artículos obtenidos fueron 1004, después de aplicar el filtro de 5 años los artículos seleccionados fueron 273, al leer el título y los resúmenes se restaron 185 obteniendo 88 artículos, se eliminaron 11 artículos duplicados quedándonos 77 artículos, aplicando criterios de inclusión y exclusión se removieron 68 artículos, tomando en cuenta 9 artículos para la revisión de la literatura. En la figura 1 se ilustra el diagrama de flujo de selección de artículos.

\section{Criterios de inclusión}

-Artículos en inglés disponibles en las bases digitales de Pubmed, Scopus y Web of Science.

-Disponibilidad de texto completo

- Artículos hasta 5 años atrás (2016-2020- noviembre).

- Artículos que hagan la comparación entre los dos tratamientos (Peróxido de Hidrógeno y Peróxido de Carbamida)

- Estudios realizados en pacientes humanos con piezas sanas.

\section{Criterios de exclusión}

-Artículos que combinan tratamientos de aclaramiento con otros productos.

- Artículos que se repitan en las otras bases digitales

- Artículos que incluyen estudios con luz LED violeta

- Estudios realizados en piezas endodonciadas. 
${ }^{1}$ Figura 1. Diagrama de flujo de selección de artículos.

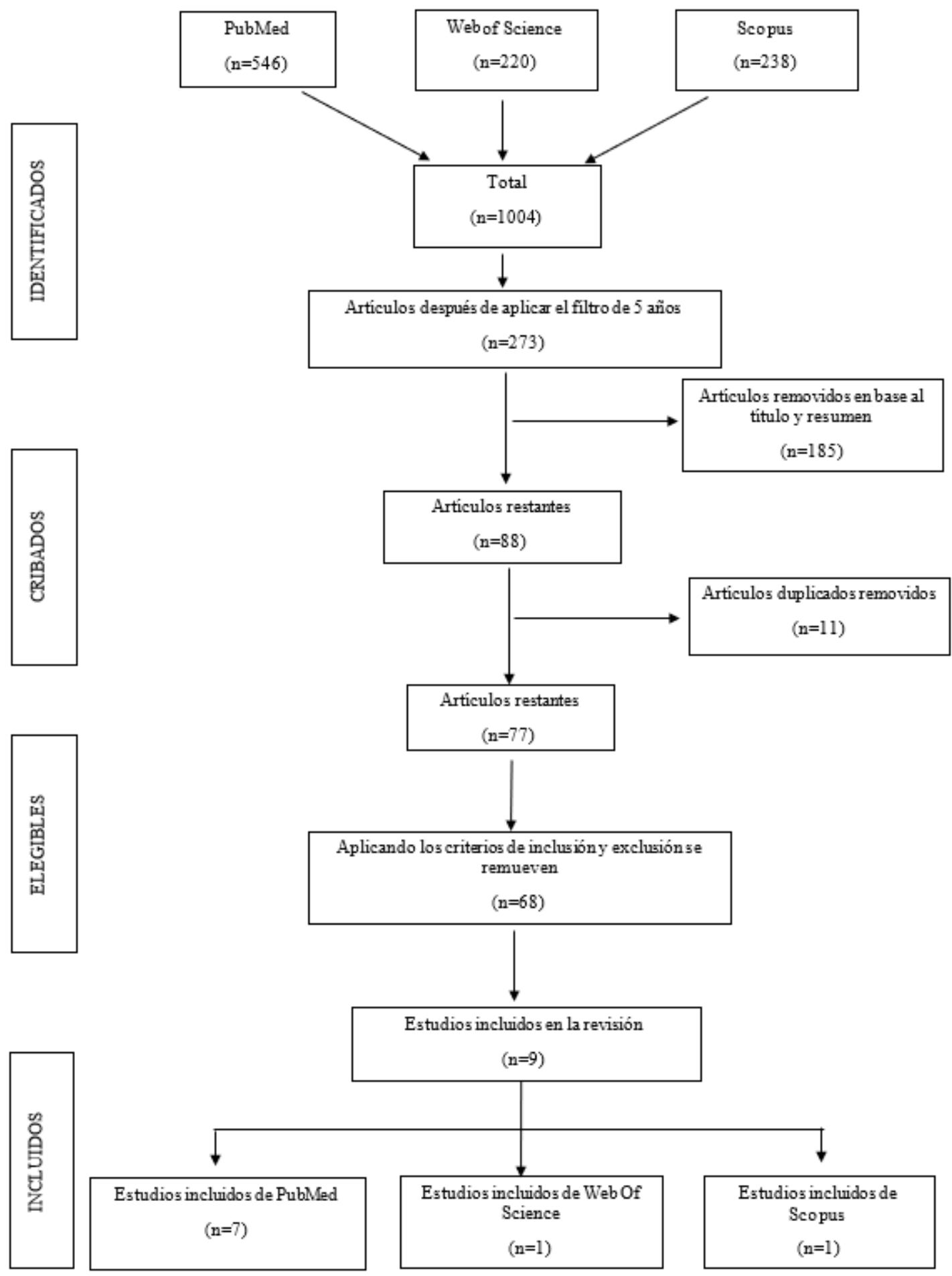

Fuente: Autores.

\section{Resultados}

Los resultados obtenidos son heterogéneos ya que los estudios incluidos no realizaron el mismo protocolo, hubo variación en el número y tiempo de aplicaciones, número de días y porcentaje del producto utilizado, así como el tiempo de evaluación del color y sensibilidad, los resultados de los estudios analizados en esta revisión se muestran en la Tabla 1.

${ }^{1}$ Se puede observar que se incluyo 9 artículos en esta revisión, de los cuales su mayoría se obtuvo de la base de datos PubMed, seguidos por Web of Science y Scopus. 


\begin{tabular}{|c|c|c|c|c|c|c|}
\hline Autores & $\begin{array}{l}\text { Tipo de } \\
\text { estudio }\end{array}$ & $\begin{array}{c}\text {-Número de pacientes } \\
\% \mathbf{P H}\end{array}$ & $\begin{array}{l}\text {-Número de pacientes } \\
\% \text { PC }\end{array}$ & Método Aplicación PH - PC & Métodos de evaluación del color & $\begin{array}{l}\text { Métodos de evaluación } \\
\text { de sensibilidad }\end{array}$ \\
\hline $\begin{array}{l}\text { Kothari S. y cols. } \\
\text { (Kothari et al., 2020) }\end{array}$ & ECA & $\begin{array}{c}-35 \text {, al final } 26 \text { pacientes } \\
37.5 \%\end{array}$ & $\begin{array}{l}-35 \text {, al final } 32 \text { pacientes } \\
10 \%\end{array}$ & $\begin{array}{c}\text { PH: } 8 \text { minutos por } 3 \text { veces. } \\
\text { PC: Bandeja personalizada, } 8 \text { horas } \\
\text { por } 14 \text { días. }\end{array}$ & $\begin{array}{c}\text { Guía de colores (Vitapan } \\
\text { Classical) y espectrofotómetro } \\
\text { (Easyshade) }\end{array}$ & n.r. \\
\hline $\begin{array}{l}\text { Bernardon JK. y cols } \\
\text { (Bernardon et al., 2015) }\end{array}$ & $\mathrm{EC}$ & $\begin{array}{c}-30 \text { (boca dividida) } \\
\text { Mx: } \\
\text { Derecha: } 35 \%, \\
\text { Izquierda: } 35 \%+\mathrm{Ca}\end{array}$ & $\begin{array}{c}-30 \text { (boca dividida) } \\
\text { Mn: } \\
\text { Derecha:10\%, Izquierda:22\% }\end{array}$ & $\begin{array}{l}\text { PH: } 40 \text { minutos por } 6 \text { semanas } \\
\text { máximo. } \\
\text { PC: Bandeja personalizada, } 2 \text { horas } \\
\text { al día por } 6 \text { semanas máximo. }\end{array}$ & Espectrofotómetro (Easyshade) & n.r. \\
\hline $\begin{array}{l}\text { Peixoto AC. y cols. } \\
\text { (Peixoto et al., 2018) }\end{array}$ & ECA & $\begin{array}{l}-20 \\
35 \%\end{array}$ & $\begin{array}{l}-20 \\
-37 \%\end{array}$ & $\begin{array}{c}\text { PH: } 3 \text { aplicaciones de } 15 \text { minutos } \\
\text { por } 2 \text { sesiones. } \\
\text { PC: } 2 \text { sesiones de } 40 \text { minutos. }\end{array}$ & Espectrofotómetro (Easyshade) & EVA y ECV \\
\hline $\begin{array}{l}\text { Ciavoi G. y cols. (Ciavoi } \\
\text { et al., 2017) }\end{array}$ & ECA & $\begin{array}{l}-50, \text { al final } 41 \\
-50 \text {, al final } 43 \\
35 \%-40 \%\end{array}$ & $\begin{array}{c}-50 \text {, al final } 39 \\
-43 \text {, al final } 38 \\
10 \%-20 \%\end{array}$ & $\begin{array}{l}\text { PH: En cubeta durante } 20 \text { minutos. } \\
\text { PC: Recomendación del fabricante. }\end{array}$ & n.r. & EVA modificada \\
\hline $\begin{array}{l}\text { Carlos NR. y cols. } \\
\text { (Carlos et al., 2017) }\end{array}$ & ECA & $\begin{array}{c}-50 \text { dividido en } 2 \text { grupos } \\
\text { PH 10\% (bandejas precargadas) } \\
\text { PH 9,5\% (bandejas personalizadas) }\end{array}$ & $\begin{array}{c}-25 \\
10 \% \text { (bandejas personalizadas) }\end{array}$ & $\begin{array}{c}\text { PH: } 30 \text { minutos diarios por } 14 \text { días. } \\
\text { PC: } 8 \text { horas durante la noche por } 14 \\
\text { días. }\end{array}$ & $\begin{array}{l}\text { Espectrofotómetro (Easyshade) y } \\
\text { Guia de colores (Vita Classical } \\
\text { Scale), Escala Vita 3D Master }\end{array}$ & EVA* \\
\hline $\begin{array}{l}\text { López Darriba I. y cols. } \\
\text { (López Darriba et al., } \\
\text { 2017) } \\
\end{array}$ & ECA & $\begin{array}{l}-20 \\
-20 \\
7.5 \% \\
\end{array}$ & $\begin{array}{l}-20 \\
-20 \\
10 \% \\
\end{array}$ & $\begin{array}{c}\text { PH y PC: } 1 \text { hora al día y durante la } \\
\text { noche por } 14 \text { días. }\end{array}$ & $\begin{array}{l}\text { Espectrofotómetro (Easyshade), } \\
\text { Vita Classical y Vita 3-D Master }\end{array}$ & Cuestionario de 4 puntos \\
\hline $\begin{array}{l}\text { Aka B. y cols. (Aka \& } \\
\text { Celik, 2017) }\end{array}$ & ECA & $\begin{array}{l}-31 \\
6 \%\end{array}$ & $\begin{array}{c}-30 \text { pacientes, subdividieron los } \\
\text { dientes en } 3 \text { grupos: Claro, medio } \\
\text { oscuro y oscuro. } \\
10 \%\end{array}$ & $\begin{array}{l}\text { PH: Cubetas precargadas } 1 \text { hora } \\
\text { diaria durante } 14 \text { días. } \\
\text { PC: } 8 \text { a } 10 \text { horas al día durante } 14 \\
\text { días. }\end{array}$ & $\begin{array}{l}\text { Espectrofotómetro (Spectro } \\
\text { Shade) y Guía de colores }\end{array}$ & Escala de 7 puntos \\
\hline $\begin{array}{l}\text { Radi RE. y cols. (Radi et } \\
\text { al., 2018) }\end{array}$ & ECA & $\begin{array}{l}-12 \\
40 \%\end{array}$ & $\begin{array}{l}-12 \\
16 \%\end{array}$ & $\begin{array}{l}\text { PH: } 2 \text { aplicaciones de } 15 \text { minutos. } \\
\text { PC: Bandejas prefabricadas, } 1 \text { hora } \\
\text { durante la noche por } 4 \text { noches. }\end{array}$ & Espectrofotómetro (Easyshade) & $\mathrm{EVA}^{* *}$ \\
\hline $\begin{array}{l}\text { Nie J. y cols. (Nie et al., } \\
\text { 2017) }\end{array}$ & ECA & $\begin{array}{l}-20 \\
38 \%\end{array}$ & $\begin{array}{l}-20 \\
10 \%\end{array}$ & $\begin{array}{c}\text { PH: } 3 \text { aplicaciones de } 15 \text { minutos. } \\
\text { PC: Bandejas personalizadas, } 8 \\
\text { horas por } 12 \text { noches. }\end{array}$ & $\begin{array}{l}\text { Espectrofotómetro (Crystaleye } \\
\text { Spectrophotometer) }\end{array}$ & $\begin{array}{c}\text { Escala ordinal Likert y } \\
\text { EVA }^{* * *}\end{array}$ \\
\hline
\end{tabular}


Research, Society and Development, v. 10, n. 5, e24210515110, 2021

(CC BY 4.0) | ISSN 2525-3409 | DOI: http://dx.doi.org/10.33448/rsd-v10i5.15110

\begin{tabular}{|c|c|c|}
\hline Autores & Resultado Color & Resultado Sensibilidad \\
\hline $\begin{array}{l}\text { Kothari S. y cols. } \\
\text { (Kothari et al., 2020) }\end{array}$ & La diferencia no fue significativa entre los dos grupos. & n.r. \\
\hline $\begin{array}{l}\text { Bernardon JK. y cols. } \\
\text { (Bernardon et al., 2015) }\end{array}$ & Todos los agentes aclaradores fueron efectivos. & n.r. \\
\hline $\begin{array}{l}\text { Peixoto AC. y cols. } \\
\text { (Peixoto et al., 2018) }\end{array}$ & Mayor significancia para $\mathrm{PH}$. & Menor sensibilidad para PC. \\
\hline $\begin{array}{l}\text { Ciavoi G. y cols. (Ciavoi } \\
\text { et al., 2017) }\end{array}$ & n.r. & Mayor sensibilidad para PC en comparación para PH. \\
\hline 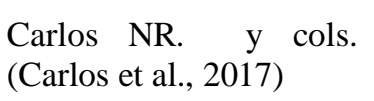 & $\begin{array}{l}\text { No se observaron diferencias significativas } \\
\text { entre los grupos o entre los períodos de tiempo. }\end{array}$ & $\begin{array}{l}\text { Se informó como leve, independientemente de la } \\
\text { concentración del agente aclarador y el tiempo de aplicación. }\end{array}$ \\
\hline $\begin{array}{l}\text { López Darriba I. y cols. } \\
\text { (López Darriba et al., } \\
2017 \text { ) }\end{array}$ & $\begin{array}{l}\text { Después de dos semanas los mismos productos } \\
\text { aplicados durante la noche fueron más efectivos que } \\
\text { aplicados } 1 \text { hora al día. }\end{array}$ & PC mostró menos sensibilidad que $\mathrm{PH}$. \\
\hline $\begin{array}{l}\text { Aka B. y cols. } 2017 \text { (Aka } \\
\& \text { Celik, 2017) }\end{array}$ & $\begin{array}{l}\text { No se observaron diferencias significativas } \\
\text { entre los grupos o entre los períodos de tiempo. }\end{array}$ & No se observaron diferencias significativas. \\
\hline $\begin{array}{l}\text { Radi RE. y cols. } 2018 \\
\text { (Radi et al., 2018) }\end{array}$ & PH y PC son igualmente efectivos. & Mayor nivel de sensibilidad con PH. \\
\hline $\begin{array}{l}\text { Nie J. y cols. } 2016 \text { (Nie et } \\
\text { al., 2017) }\end{array}$ & PC fue más eficaz. & PC menor sensibilidad que PH. \\
\hline \multicolumn{3}{|c|}{ 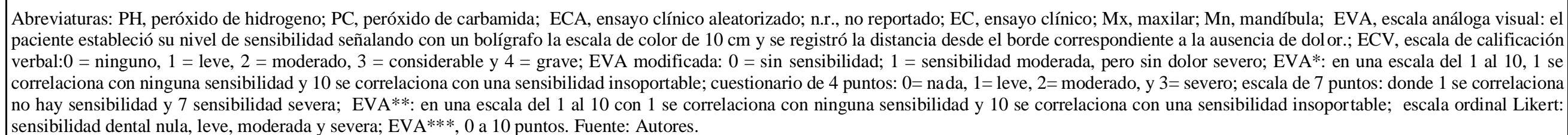 } \\
\hline
\end{tabular}




\section{Discusión}

La eficacia del clareamiento se determina principalmente por el grado de cambio de color del diente evaluado al inicio y en periodos posteriores al tratamiento, de acuerdo a la Asociación Dental Americana (ADA), los agentes aclarantes resultan eficaces cuando el color resultante es al menos 4 tonos más claro con una guía de colores o es de 3 unidades más bajo o más alto con el sistema CIELab (Bernardon et al., 2015).

De acuerdo a los estudios analizados se encontró que todos los agentes aclaradores mejoran el aspecto del color de las estructuras dentales. Peixoto y Carlos en sus resultados observaron que el PH produce un mejor cambio de color, aunque Carlos reporta que el PH y el PC son igualmente efectivos al evaluar el color con el espectrofotómetro (Moradas 2017) (Carlos et al., 2017). En contraste con López y Aka que en sus estudios aplican PH 7.5\% y 6\%, respectivamente, porcentajes más bajos en comparación con el resto de estudios, los resultados obtenidos son los mismo tanto para el PH como para el PC (López Darriba et al., 2017) (Aka \& Celik, 2017). Nie aplica PH al 38\%, encontró que el PC es mejor agente aclarador que el PH (LuqueMartinez et al., 2016). En cuanto a los estudios de Kotharia, Radi y Bernardon no encontraron diferencias entre PC y PH (Kothari et al., 2020) (Radi et al., 2018) (Bernardon et al., 2015). Cabe recalcar que Ciaovi en su estudio no evalúa el color (Ciavoi et al., 2017).

La evaluación del color se realiza en base a una guía de colores dentales estandarizada organizada según valores, es decir, el número de tonos más claros, este enfoque de evaluación de la eficacia del clareamiento es algo subjetivo y no cuantificable; sin embargo, existen métodos con mediciones objetivas, precisas y cuantificables como los espectrofotómetros que son aparatos utilizados en la medición del color de un objeto a través de su longitud de onda reflejada (Eachempati et al., 2018).

Todos los artículos analizados evalúan el color mediante el espectrofotómetro(Kothari et al., 2020)(Bernardon et al., 2015)(Peixoto et al., 2018)(Carlos et al., 2017)(López Darriba et al., 2017)(Aka \& Celik, 2017)(Radi et al., 2018)(Nie et al., 2017). Carlos, en su estudio evalúa el color con espectrofotómetro (Easyshade), guia de colores (Vita Classical Scale) y escala Vita 3D Master y sus resultados varían de acuerdo a el método de evaluación aplicado; según la guía de colores y la escala Vita 3D Master se encontró peores resultados para el PH y con el uso del espectrofotómetro no se observaron diferencias entre los grupos o entre los períodos de tiempo (Carlos et al., 2017).

La sensibilidad reportada por pacientes después de procedimientos de clareamiento dental está relacionada con procesos inflamatorios inducidos por la presencia de peróxido y sus productos en la cámara pulpar, reduciendo la proliferación, metabolismo y viabilidad de las células pulpares, y comprometiendo la capacidad de reparación de la pulpa (Peixoto et al., 2018).

La sensibilidad dental es producida por todos los agentes aclarantes, pero en diferentes niveles. Peixoto observó que el PC produce menor sensibilidad durante el tratamiento; sin embargo, después de 24 horas no hubo diferencias entre PH y PC; lo que concuerda con los resultados obtenidos por Nie y López, mayor sensibilidad para el PH inmediatamente después del tratamiento, Radi concluyó que el PH al 16\% generaba mayor sensibilidad (Peixoto et al., 2018)(Nie et al., 2017)(López Darriba et al., 2017)(Radi et al., 2018). En contraste con esto, Ciavoi obtuvo que el PC aplicado al 10\% o 20\% generaba mayor sensibilidad(Ciavoi et al., 2017). Carlos encontró una sensibilidad leve independientemente del agente utilizado (Carlos et al., 2017). En cuanto al estudio realizado por Aka obtuvo que tanto el PH como PC fueron iguales en cuanto a la sensibilidad(Aka \& Celik, 2017). Kothari y Bernardon no evalúan la sensibilidad en sus estudios(Kothari et al., 2020)(Bernardon et al., 2015).

Con respecto al nivel de sensibilidad dental, los datos sobre el PC y PH en los estudios analizados fueron evaluados en su mayoría con EVA (Escala Visual Análoga) (Nie et al., 2017)(Peixoto et al., 2018) (Carlos et al., 2017) (Radi et al., 2018)., EVA modificado(Ciavoi et al., 2017), seguido por ECV (Escala de Calificación Verbal)(Peixoto et al., 2018), Escala Ordinal 
Likert(Nie et al., 2017), Escala de 7 puntos (Aka \& Celik, 2017) y por último un Cuestionario de 4 puntos(López Darriba et al., 2017), donde se encontró que el PC presenta niveles de sensibilidad dental cercanos a 1 en la mayoría de los artículos analizados.

\section{Conclusión}

De acuerdo a los resultados analizados, se concluyó que tanto el peróxido de hidrógeno como el peróxido de carbamida son efectivos para producir un cambio de color de los dientes. En los dientes más oscuros el tratamiento es más efectivo independientemente de agente aclarante utilizado. La eficacia del tratamiento depende del tiempo de aplicación y la sensibilidad está relacionada con la concentración del agente aclarante. En base a la investigación realizada se puede concluir que el peróxido de carbamida produce menor sensibilidad en comparación con el peróxido de hidrógeno.

Se recomienda que las futuras investigaciones incluyan estudios con protocolos, porcentajes con un rango de edades homogéneos para comparar la eficacia del aclareamiento dental con PC y PH. Además, el tiempo de evaluación del color en los artículos estudiados fue variado, por lo que se sugiere que las evaluaciones sean a corto, mediano y largo plazo. Por otro lado, para investigaciones acerca de la sensibilidad dental se sugiere que los estudios incluidos tengan el mismo método de evaluación.

\section{Referencias}

Ahrari, F., Akbari, M., Mohammadipour, H. S., Fallahrastegar, A., \& Sekandari, S. (2020). The efficacy and complications of several bleaching techniques in patients after fixed orthodontic therapy. A randomized clinical trial. Swiss Dental Journal, 130(6), 493-501.

Aka, B., \& Celik, E. U. (2017). Evaluation of the Efficacy and Color Stability of Two Different At-Home Bleaching Systems on Teeth of Different Shades: A Randomized Controlled Clinical Trial. Journal of Esthetic and Restorative Dentistry, 29(5), 325-338. https://doi.org/10.1111/jerd.12296

Alkahtani, R., Stone, S., German, M., \& Waterhouse, P. (2020). A review on dental whitening. Journal of Dentistry, 100, 103423. https://doi.org/10.1016/j.jdent.2020.103423

Alomar, A., \& Manubens, E. (2015). Bleaching agents. European Handbook of Dermatological Treatments, 4, 1421-1426. https://doi.org/10.1007/978-3-66245139-7_138

Alqahtani, M. Q. (2014). Tooth-bleaching procedures and their controversial effects: A literature review. Saudi Dental Journal, 26(2), 33-46. https://doi.org/10.1016/j.sdentj.2014.02.002

Bernardon, J. K., Ferrari, P., Baratieri, L. N., \& Rauber, G. B. (2015). Comparison of treatment time versus patient satisfaction in at-home and in-office tooth bleaching therapy. Journal of Prosthetic Dentistry, 114(6), 826-830. https://doi.org/10.1016/j.prosdent.2015.05.014

Carlos, N. R., Bridi, E. C., Amaral, F. L. B., França, F. M. G., Turssi, C. P., \& Basting, R. T. (2017). Efficacy of home-use bleaching agents delivered in customized or prefilled disposable trays: A randomized clinical trial. Operative Dentistry, 42(1), 30-40. https://doi.org/10.2341/15-315-C

Ciavoi, G., Bechir, A., Suciu, M., Bechir, E. S., Doda, L., Olteanu, C., \& Dascalu, I. T. (2017). The role of peroxide based bleaching agents in the induction of tooth sensitivity. Revista de Chimie, 68(5), 911-915. https://doi.org/10.37358/rc.17.5.5579

Correa, A. C., Santana, T. R., Nahsan, F. P., Loguercio, A. D., \& Faria-E-Silva, A. L. (2016). The impact of a customized tray on in-office bleaching tooth sensitivity: A randomized clinical trial. Operative Dentistry, 4l(1), 15-22. https://doi.org/10.2341/15-029-C

Eachempati, P., Kumbargere Nagraj, S., Kiran Kumar Krishanappa, S., Gupta, P., \& Yaylali, I. E. (2018). Home-based chemically-induced whitening (bleaching) of teeth in adults. Cochrane Database of Systematic Reviews, 2018(12). https://doi.org/10.1002/14651858.CD006202.pub2

Eimar, H., Siciliano, R., Abdallah, M. N., Nader, S. A., Amin, W. M., Martinez, P. P., Celemin, A., Cerruti, M., \& Tamimi, F. (2012). Hydrogen peroxide whitens teeth by oxidizing the organic structure. Journal of Dentistry, 40(2), e25-e33. https://doi.org/10.1016/j.jdent.2012.08.008

Jadhav, R., \& Mattigatti, S. (2020). "Critical evaluatiion of effiicacy, rebound effect and sensiitiiviity of in-offiice vs at-home bleachiing techniiques: An in Viivo Study.” Journal of Critical Reviews, 7(12), 769-775. https://doi.org/10.31838/jcr.07.12.137

Kothari, S., Jum'ah, A. A., Gray, A. R., M. Lyons, K., Yap, M., \& Brunton, P. A. (2020). A randomized clinical trial investigating three vital tooth bleaching protocols and associated efficacy, effectiveness and participants' satisfaction. Journal of Dentistry, 95(November 2019), 103322. https://doi.org/10.1016/j.jdent.2020.103322

López Darriba, I., Novoa, L., \& De la Peña, V. A. (2017). Efficacy of different protocols for at-home bleaching : A randomized clinical trial. Am J Dent, January 2018.

Luque-Martinez, I., Reis, A., Schroeder, M., Muñoz, M. A., Loguercio, A. D., Masterson, D., \& Maia, L. C. (2016). Comparison of efficacy of tray-delivered carbamide and hydrogen peroxide for at-home bleaching: a systematic review and meta-analysis. Clinical Oral Investigations, 20 (7), 1419-1433. https://doi.org/10.1007/s00784-016-1863-7 
Mailart, M. C., Sakassegawa, P. A., Torres, C. R. G., Palo, R. M., \& Borges, A. B. (2020). Assessment of peroxide in saliva during and after at-home bleaching with $10 \%$ carbamide and hydrogen peroxide gels: A clinical crossover trial. Operative Dentistry, 45(4), 368-376. https://doi.org/10.2341/19-127-C

Moradas M. (2017). ¿qué material y técnica seleccionamos a la hora de realizar un blanqueamiento dental y por qué? Protocolo para evitar hipersensibilidad dental posterior. Avances En Odontoestomatologia, 33(3), 103-112.

Mounika, A., Mandava, J., Roopesh, B., \& Karri, G. (2018). Clinical evaluation of color change and tooth sensitivity with in-office and home bleaching treatments. Indian Journal of Dental Research, 29(4), 423-427. https://doi.org/10.4103/ijdr.IJDR_688_16

Nie, J., Tian, F. C., Wang, Z. H., Yap, A. U., \& Wang, X. Y. (2017). Comparison of efficacy and outcome satisfaction between in-office and home teeth bleaching in Chinese patients. Journal of Oral Science, 59(4), 527-532. https://doi.org/10.2334/josnusd.16-0636

Pan, Q., \& Westland, S. (2018). Tooth color and whitening - digital technologies. Journal of Dentistry, 74(March), S42-S46. https://doi.org/10.1016/j.jdent.2018.04.023

Peixoto, A. C., Vaez, S. C., Pereira, N. A. de R., Santana, C. N. da S., Soares, K. D. A., Romão, A. C. T. R., Ferreira, L. F., Martins-Filho, P. R. S., \& Faria-ESilva, A. L. (2018). High-concentration carbamide peroxide can reduce the sensitivity caused by in-office tooth bleaching: a single-blinded randomized controlled trial. Journal of Applied Oral Science : Revista FOB, 26, e20170573. https://doi.org/10.1590/1678-7757-2017-0573

Pereira, A., Shitsuka, D., Parreira, F., \& Shitsuka, R. (2018). Método Qualitativo, Quantitativo ou Quali-Quanti. In Metodologia da Pesquisa Científica. https://repositorio.ufsm.br/bitstream/handle/1/15824/Lic_Computacao_Metodologia-Pesquisa-Cientifica.pdf?sequence=1. Acesso em: 28 março 2020.

Radi, R. E., Abdelwahab, S., \& Abdelaleem, N. (2018). Comparative Study between At-Home \& In-Office Bleaching on Color Stability and Teeth Sensitivity in Makkah City, Saudi Arabia. International Journal of Health Sciences \& Research (Www.Ijhsr.Org), 8(2), 89-100. www.ijhsr.org

Rodrigues, J. L., Rocha, P. S., Pardim, S. L. de S., Machado, A. C. V., Faria-e-Silva, A. L., \& Seraidarian, P. I. (2018). Association between in-office and athome tooth bleaching: A single blind randomized clinical trial. Brazilian Dental Journal, 29(2), 133-139. https://doi.org/10.1590/0103-6440201801726

Rodríguez-Martínez, J., Valiente, M., \& Sánchez-Martín, M. J. (2019). Tooth whitening: From the established treatments to novel approaches to prevent side effects. Journal of Esthetic and Restorative Dentistry, 31(5), 431-440. https://doi.org/10.1111/jerd.12519

Schmelling, M. (2016). Color Selection and Reproduction in Dentistry. Part 3/ Visual and Instrumental Shade Matching. ODOVTOS-Int. J. Dental., 1(JANUARY), 23-32. https://www.scielo.sa.cr/pdf/odovtos/v19n1/2215-3411-odovtos-19-01-00023.pdf

Solís Cessa, E. (2018). Aclaramiento dental: revisión de la literatura y presentación de un caso clínico. Dental clearance: review of the literature and case report. Revista ADM, 75(1), 9-25.

Sulieman, M., Macdonald, E., Rees, J. s., Newcombe, R. g., \& Addy, M. (2006). Tooth Bleaching by Different Concentrations of Carbamide Peroxide and Hydrogen Peroxide Whitening Strips: An In Vitro Study. Journal of Esthetic and Restorative Dentistry, 18(2), 93-100. https://doi.org/10.2310/6130.2006.00016_1.x

Vaz, M. M., Lopes, L. G., Cardoso, P. C., de Souza, J. B., Batista, A. C., Costa, N. L., Torres, É. M., \& Estrela, C. (2016). Inflammatory response of human dental pulp to at-home and in-office tooth bleaching. Journal of Applied Oral Science, 24(5), 509-517. https://doi.org/10.1590/1678-775720160137

Zanolla, J., Marques, A. B. C., da Costa, D. C., de Souza, A. S., \& Coutinho, M. (2017). Influence of tooth bleaching on dental enamel microhardness: a systematic review and meta-analysis. Australian Dental Journal, 62(3), 276-282. https://doi.org/10.1111/adj.12494 